\title{
Experimental validation of A-mode ultrasound acquisition system for computer assisted orthopaedic surgery
}

\author{
Danilo De Lorenzo*a, Elena De Momi ${ }^{\mathrm{a}}$, Elisa Beretta ${ }^{\mathrm{a}}$, Pietro Cerveri ${ }^{\mathrm{a}}$, Franco Perona ${ }^{\mathrm{b}}$, Giancarlo \\ Ferrigno $^{\mathrm{a}}$ \\ ${ }^{a}$ NearLab, Bioengineering Department, Politecnico di Milano, Via Golgi 39, 20133 Milan, Italy \\ 'b.R.C.C.S. Istituto Ortopedico Galeazzi - GSD, via R. Galeazzi 4, 20161 Milan, Italy
}

\begin{abstract}
Computer Assisted Orthopaedic Surgery (CAOS) systems improve the results and the standardization of surgical interventions. Anatomical landmarks and bone surface detection is straightforward to either register the surgical space with the pre-operative imaging space and to compute biomechanical parameters for prosthesis alignment.

Surface points acquisition increases the intervention invasiveness and can be influenced by the soft tissue layer interposition (7-15mm localization errors).

This study is aimed at evaluating the accuracy of a custom-made A-mode ultrasound (US) system for non invasive detection of anatomical landmarks and surfaces. A-mode solutions eliminate the necessity of US images segmentation, offers real-time signal processing and requires less invasive equipment. The system consists in a single transducer US probe optically tracked, a pulser/receiver and an FPGA-based board, which is responsible for logic control command generation and for real-time signal processing and three custom-made board (signal acquisition, blanking and synchronization).

We propose a new calibration method of the US system. The experimental validation was then performed measuring the length of known-shape polymethylmethacrylate boxes filled with pure water and acquiring bone surface points on a bovine bone phantom covered with soft-tissue mimicking materials. Measurement errors were computed through MR and CT images acquisitions of the phantom. Points acquisition on bone surface with the US system demonstrated lower errors $(1.2 \mathrm{~mm})$ than standard pointer acquisition $(4.2 \mathrm{~mm})$.
\end{abstract}

Keywords: Ultrasound, Computer Assisted Orthopedic Surgery, A-mode calibration

\section{INTRODUCTION}

Computer assisted orthopaedic surgery (CAOS) systems for total hip and total knee replacements (THR and TKR, respectively) have proven to improve the results of surgical intervention in terms of prostheses alignment, soft tissue balancing and reducing deviations from best practice and eliminating outliers in components alignment ${ }^{1-3}$. Also, it allows the standardization and, thus the optimization, of the surgical workflow.

In CAOS systems detecting anatomical landmarks and bone surface is straightforward in order to either register the surgical space with the pre-operative imaging space, if the images exist, and to detect anatomical landmarks used for the computation of biomechanical points for determining optimal prostheses size, position and orientation ${ }^{3}$. Bone surfaces have to be detected in order to refine the registration with the medical images space or in order to morph a statistical model of the bone $e^{4-6}$ in order to make it patient specific, so to accurately plan the best choice for the implant size.

During computer assisted interventions, the points of interest can be acquired with tracked pointers (equipped with optical or electromagnetic markers ${ }^{6}$ ).

*danilo.delorenzo@mail.polimi.it; phone 003902 2399 3371; fax 003902 2399 3360; http://www.biomed.polimi.it/nearlab

Medical Imaging 2009: Ultrasonic Imaging and Signal Processing, edited by Stephen A. McAleavey, Jan D'hooge Proc. of SPIE Vol. 7265, 726502 - (C) 2009 SPIE · CCC code: 1605-7422/09/\$18 - doi: 10.1117/12.811736 
The procedure requires the exposure of the bone surface, thus increasing the intervention invasiveness, or the percutaneous access to those points, which worsens the points detection accuracy due to the soft tissue layer interposition. It was demonstrated that inaccuracies in anatomical points detection due to external access can raise up to $7-15 \mathrm{~mm}^{7,8}$.

Minimally invasive surgical techniques improve safety and efficacy of the surgical intervention since they diminish the risk of complications, they improve the postoperative function and longevity of prosthetic components, combined with shorter hospital stay and faster recovery for the patient ${ }^{2,3}$. Recently, ultrasound (US) based systems have been introduced in the surgical room for the accurate and mini-invasive intra-operative detection of anatomical points ${ }^{9}$. The US probes are equipped with optical markers, with a singular transducer (A-mode) or with an array of transducers (B-mode). The signals are acquired together with the trackers 3D positions. Detected surfaces are referenced to reference frames fixed to the patient ${ }^{10,11}$. Recently, US images (B-mode) were used for initializing and registering statistical shape models of the femur ${ }^{5}$ and pelvis ${ }^{4}$.

A-mode US systems for fiducial markers localization were firstly introduced in neurosurgery applications in order to detect bone landmarks for the registration of the image space with the physical space ${ }^{12-14}$. They were also used for transcutaneous palpation of points for THR procedures ${ }^{15}$ and for detecting intra-femoral bone cement and 3D reconstruction in hip revision operations ${ }^{16}$. A-mode systems avoid US images segmentation, offer real-time signal processing and require less invasive equipment.

Our study is mainly aimed at evaluating the accuracy of a custom-made A-mode US system (BONUS) for non invasive detection of anatomical landmarks and bone surfaces. A new calibration method for A-mode US system is also reported. The system was tested measuring the length of known-shape polymethylmethacrylate (PMMA) boxes, filled with pure water at given temperatures. The experimental validation was performed on a bovine bone phantom covered with softtissue mimicking materials. The gold standard for errors measurements was obtained through MR and CT images acquisitions of the phantom, which were processed and segmented, thus to obtain surface models of the structures of interest.

\section{METHODOLOGY}

\subsection{US Hardware}

A-mode US systems are used in many industrial non-destructive testing equipment for flaw detection, determination of distances and wall thickness of materials. In pulse-echo technique, a short, energy pulse is driven into the medium and different echos result from different acoustic impedances encountered by the US beam. The reflection coefficient $\mathrm{R}$ between bones and soft tissues is approximately $0.4-0.5$, so echoes corresponding to the bone/soft-tissue interface are easly identified. The distance from the transducer to the bone surface is calculated by $d=1 / 2 \mathrm{ct}$, where $d$ is the distance, $c$ is the velocity of sound, and $t$ is the Time of Flight (TOF) between the pulse generation and the detection of the echo. Typical value for sound velocity in human soft tissue varies between $1450-1630 \mathrm{~m} / \mathrm{s}^{15}$.

US signal was generated by the V110-SM unfocused US transducer (Olympus-NDT, MA, US), with 6 mm element size and $5 \mathrm{MHz}$ central frequency. The nominal near field in water is $30 \mathrm{~mm}$ and the divergence angle of the beam is $3.44^{\circ}$. Axial resolution of the probe is $0.6 \mathrm{~mm}$. The transducer is firmly anchored in an aluminium made handle (probe) (Figure 5 , right).

A pulser/receiver hardware driver (5800, Olympus Panametrics-NDT, MA, US) is used for signal generation and analog signal processing: band-pass filtering $(1 \mathrm{MHz}-20 \mathrm{MHz})$ and signal amplification $(40 \mathrm{~dB})$. The device drives the transducer with a $300 \mathrm{~V}$ main bang of $200 \mathrm{~ns}$.

A blanking board was developed for impulse artifact depression in the echo signal. An IR-photodiode-based board, was developed to synchronize the repetition frequency of the exciting impulse. In order to acquire the US RF signal an electronic acquisition board, based on the THS1041 ADC (Texas Instruments, TX, USA) and on the AD8331 variable gain amplifier (Analog Devices, MA, USA) for Time Gain Compensation (TGC) purposes was realized. The entire system is shown in Figure 5. All the electronic circuits are connected to a FPGA based board (Nexys, Digilent inc., WA, USA), which process the signal in real-time. The computed distance value is sent to a PC in real-time via USB 2.0 interface. 


\subsection{Material characterization for system accuracy evaluation}

In order to evaluate the developed system, a bone phantom with soft tissue equivalent material was built. For mimicking the behaviour of soft tissues, we used the following sealant silicones:

1. Sylgard 184 (SYL)

2. Saratoga BIANCOSAN 85275001 (SARA)

3. Bostik transparent BI 3127 MM (BOT)

4. Bostik white BI $4027 \mathrm{~B}$ (BOW)

PMMA boxes of known shape were machined by milling cutter (MODELA MDX-40, Roland DG Corp., Japan) with $0.01 \mathrm{~mm}$ precision and filled with the four types of silicone and with pure water. Boxes vary in a range of $0.03-0.1 \mathrm{~m}$ in length (see Figure 1, A). The transducer was placed perpendicularly to the PMMA side and gel was interposed between the box and the probe to assure good acoustic coupling (Figure 1, B).

A

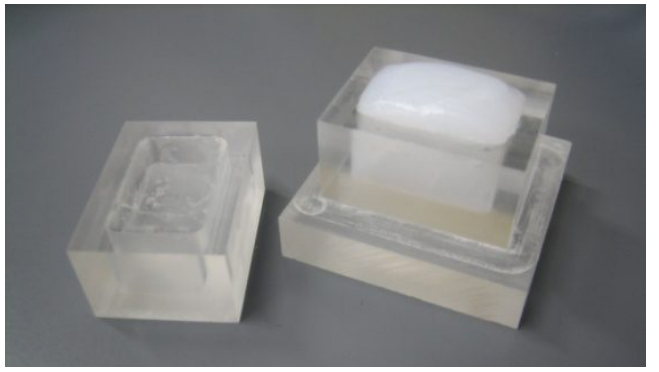

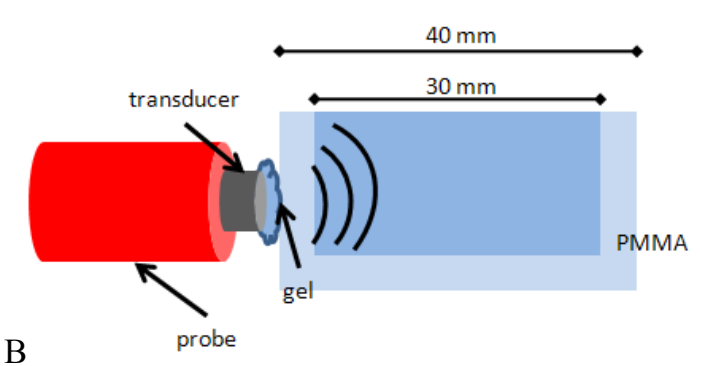

$\mathrm{B}$

Figure 1 (A): PMMA boxes filled with two different types of silicone. On the left, a 20 x $30 \mathrm{~mm}$ box filled with SYL and on the right a $20 \times 30 \mathrm{~mm}$ box filled with BOT. (B) Probe pose for signal acquisition.

In order to characterize the speed of sound in the above cited materials, four PMMA boxes of known shape were used. For each sample the TOF was detected (as described in section 2.4) and the velocity therefore computed:

$$
v_{\text {material }}=\frac{P M M A_{\text {length }} \cdot 2}{T O F}
$$

where $P M M A_{\text {length }}$ is the internal length of the PMMA box.

\subsection{Bone phantom treatment and realization}

A bovine femur was cleaned from soft tissue residuals and sterilized with bleach. The four silicones were applied on the cortical bone in order to simulate different tissues layers (e.g. cartilage, tendons, muscle and fat). In Figure 2 the different femoral areas are shown (Table 1).

Table 1 Phantom and silicone layers. The $1^{\text {st }}$ layer is the one in contact with the bone surface, the $2^{\text {nd }}$ is the one in the middle and the $3^{\text {rd }}$ the outermost.

\begin{tabular}{ccccc}
\hline Zone number & Anatomical localization & $1^{\text {st }}$ layer & $2^{\text {nd }}$ layer & $3^{\text {rd }}$ layer \\
1 & patella & BOT & BOW & BOT \\
2 & inferior diafisi, right & SARA & BOT & SYL \\
3 & inferior diafisi, medial & SARA & - & - \\
4 & inferior diafisi, left & SARA & BOT & - \\
5 & superior diafisi & BOT & SYL & - \\
6 & uppermost region & BOW & SARA & - \\
7 & condyle right & BOT & - & - \\
8 & condyle left & - & - & - \\
\hline
\end{tabular}




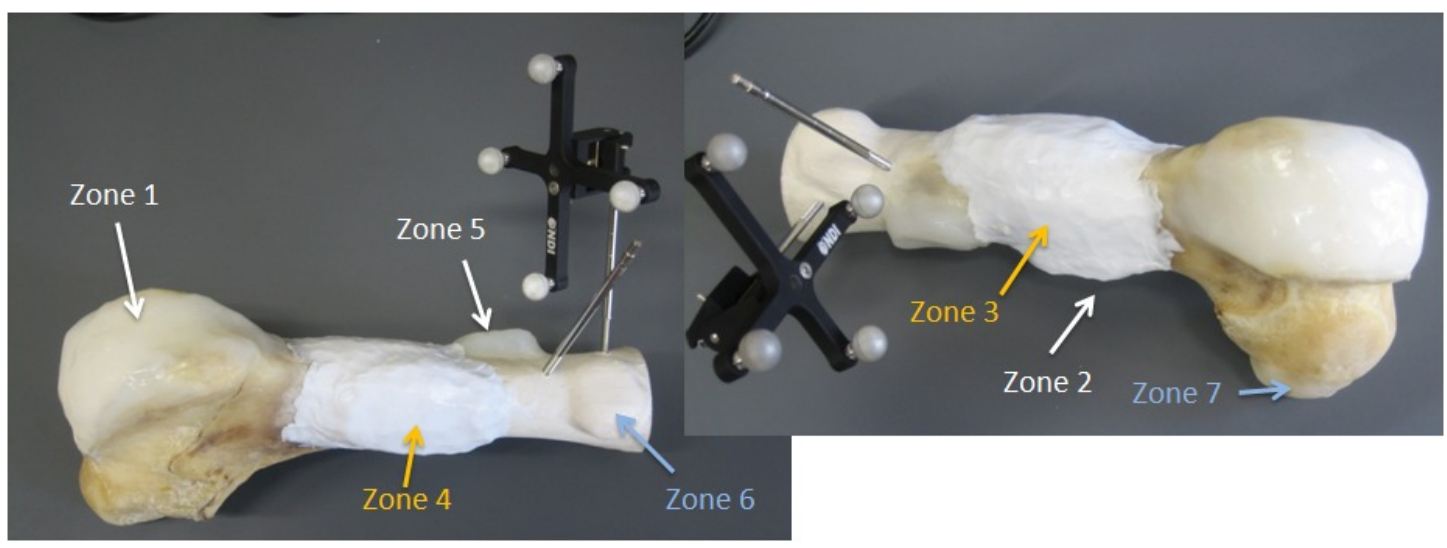

Figure 2 Bovine bone phantom with different types of silicone in different layers (zones 1..7). Femoral DRF is rigidly implanted on it.

\subsection{Signal elaboration}

In order to compute the TOF of the US beam within the medium of interest, it is necessary to detect the peaks of the acquired signal, which are due to the reflection of the US beam at the interface between materials with different acoustic impedance (Figure 3). The first deflection of the signal indicates the beam reflection. Templates of the expected signals were built, as shown in Figure 4. Signals acquired were digitally filtered (FIR, Equiripple Lowpass filter, $5^{\text {th }}$ order, bandpass frequency $4 \mathrm{MHz}$ and stop-band frequency $7 \mathrm{MHz}$ ) and then windowed with a rectangular window.

The sample corresponding to the reflection $\left(T_{\text {reflection }}\right)$ is computed as the point in which the cross-correlation between the template $(\mathrm{T})$ and the signal $(\mathrm{S})$ is maximum:

$$
T_{\text {reflection }}=\max \left\{\sum_{t=0}^{T_{f}}\left(S_{t}-t\right) \cdot\left(T_{t}\right)\right\}
$$

Where $T_{f}$ is the last sample of the signal and $t$ the time.

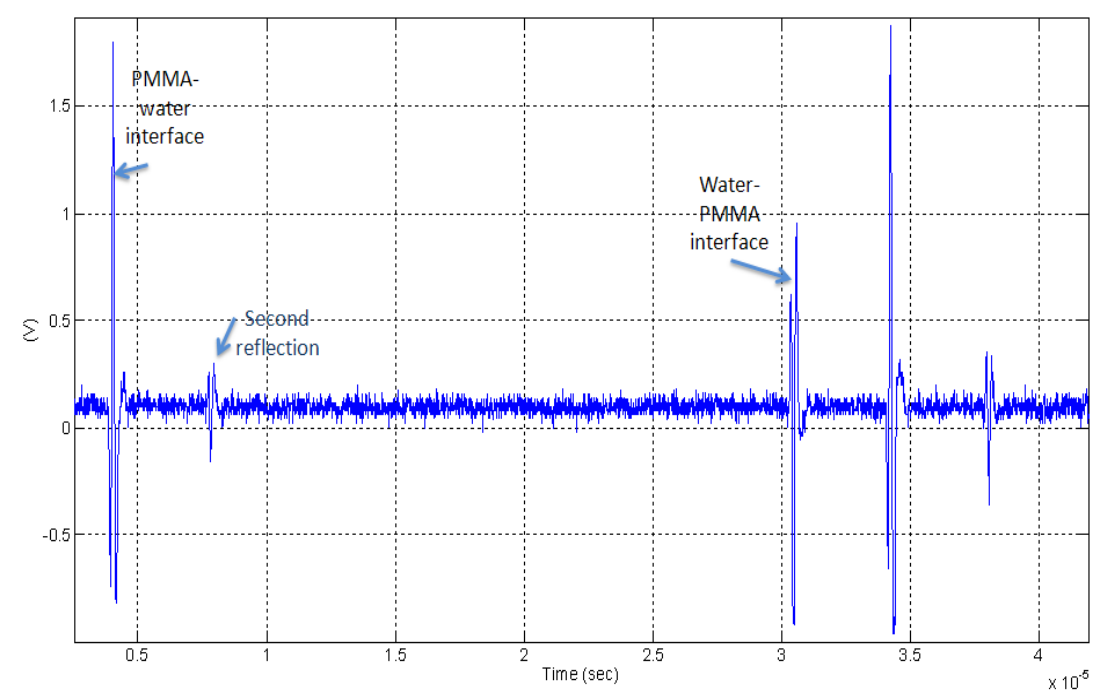

Figure 3 Example of acquired signal on the PMMA box (Figure 1) filled with pure water at $26^{\circ} \mathrm{C}$. The interfaces can be easily detected: between PMMA and water and between water and PMMA 

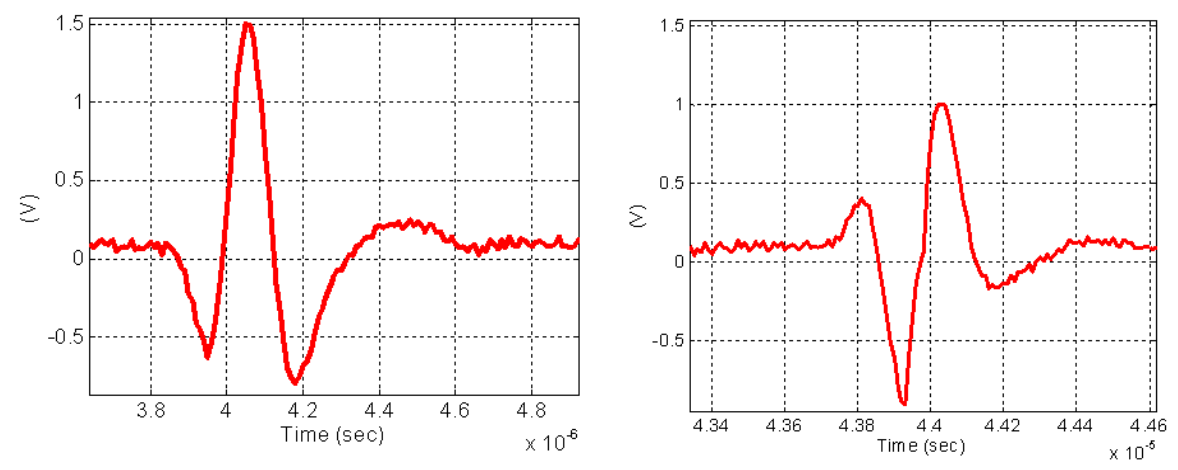

Figure 4 On the left, template for the detection of the first interface; on the right, template for the detection of the second interface. Note the phase lag between the two waves.

\subsection{Optical localization}

Dynamic Reference Frames (DRFs) of passive reflective markers were attached on the US probe and on the cortical part of the bone through cortical screws (anatomical reference frame). The pose of the bone and of the probe were both tracked with an optical localization system (VICRA, NDI, Ontario, Canada) at $20 \mathrm{~Hz}$. The 3D coordinates of surface points were detected by an optically tracked palpation probe, provided by NDI. The estimated accuracy of the pointer (in terms of Target Registration Error ${ }^{17}$ ) is $0.5 \mathrm{~mm}$.

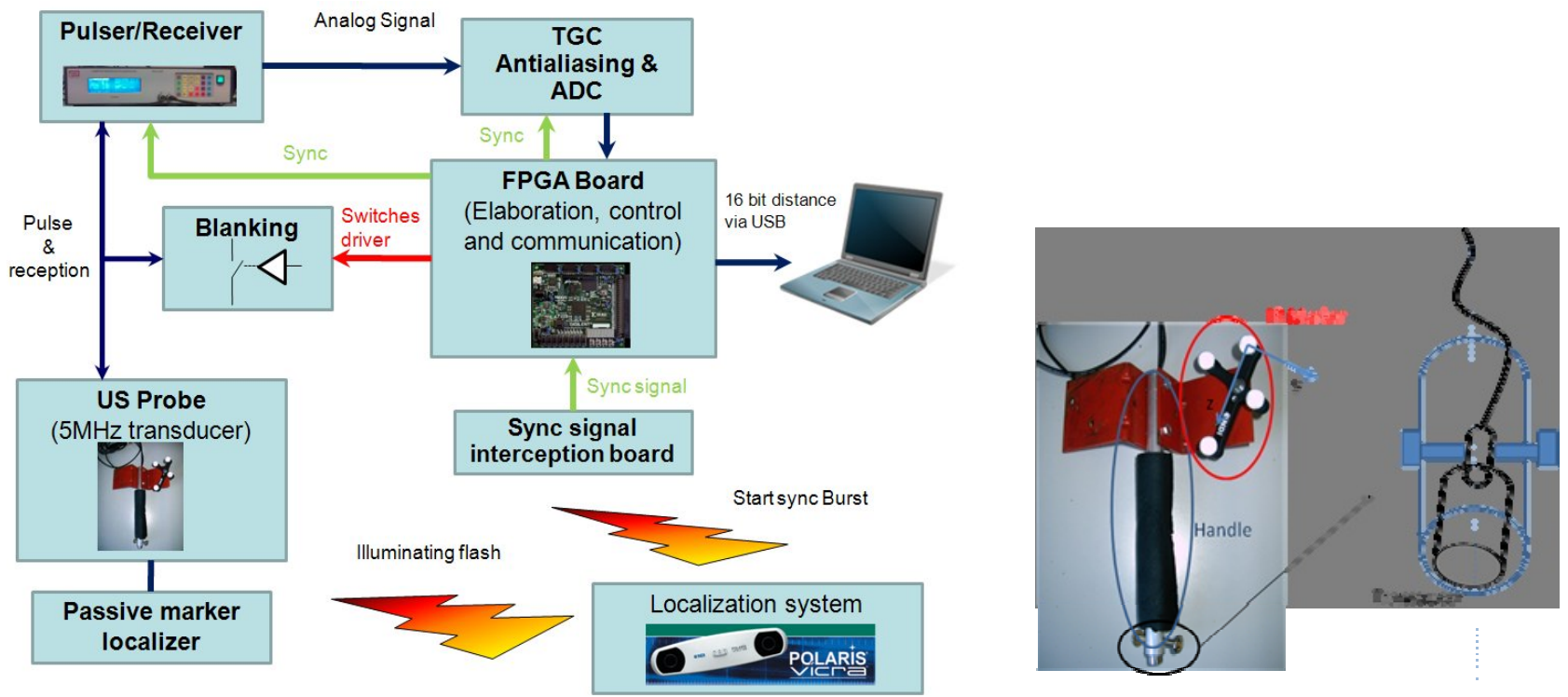

Figure 5 Block Scheme of the US system (left). The US probe (handle and transducer) with a detailed view of the transducer mounting on the handle (right).

\subsection{US probe calibration (BONUS)}

BONUS system calibration means computing the origin and the direction of the US beam with respect to the probe DRF. The US beam origin, $\boldsymbol{O}$, is defined by its spatial coordinates $\left(\mathrm{X}_{0}, \mathrm{Y}_{0}, \mathrm{Z}_{0}\right)$ and the line direction, $\boldsymbol{t}$, by its director cosines $(\alpha, \beta, \gamma)$. The point $\boldsymbol{P}$ on the reflective plane, $\pi$, used for calibration is given by: $\mathbf{P}=\mathbf{O}+d \cdot \mathbf{t}$, where $d$ is the distance from the point $\boldsymbol{O}$ to the plane $\pi$ and $\boldsymbol{t}$ is the beam direction. The plane $\pi$ equation is $(\mathbf{P}-\mathbf{Q}) \cdot \mathbf{u}=0$, where $\boldsymbol{Q}$ is the plane origin and $\mathbf{u}$ is the plane normal in the probe reference system estimated acquiring $n=50$ points on the plane.

For each pose of the probe in space, the US signal is acquired and the distance $d_{i}$ ' is computed. Also, the geometrical distance value $d_{i}$ from the origin $\boldsymbol{O}$ of the beam and the intersection point $\boldsymbol{P}$ between the plane $\pi$ and the straight line $\boldsymbol{t}$, can be computed. 


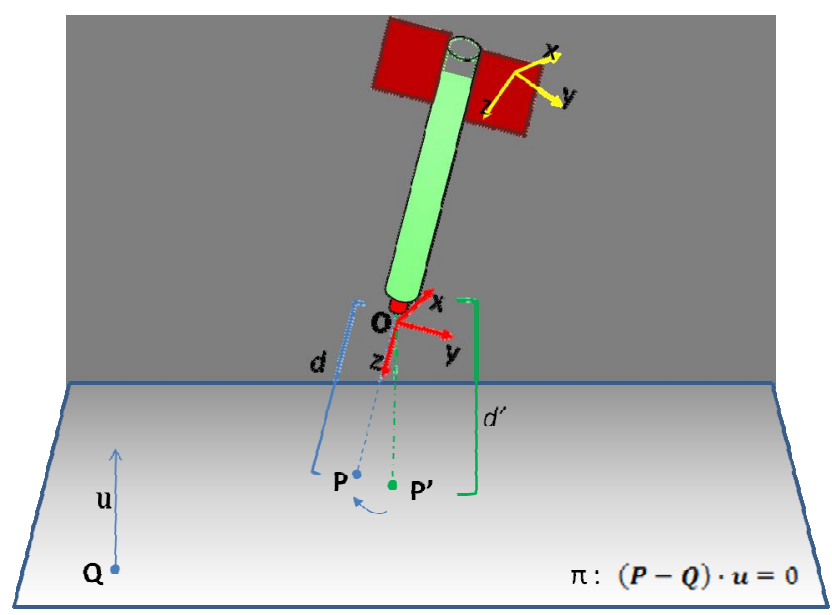

Figure 6 Schematic illustration of the calibration method.

Then, the unknown parameter $\boldsymbol{t}$ and $\boldsymbol{O}$ were computed minimizing the following cost function with Levenberg Marquardt optimization algorithm $^{18,19}$ :

$$
F=\sum_{i=1}^{N}\left(d_{i}-d_{i}^{\prime}\right)^{2}
$$

where $N$ is the number of the acquired probe poses.

The origin $\boldsymbol{O}$ and the direction $\boldsymbol{t}$ were iteratively evaluated. The acquired dataset was divided in a training set and in a testing set. The iterations were stopped when the root mean square (RMS) of the calibration residuals calculated on the testing dataset reached a minimum:

$$
\min \left\{R M S\left(d_{\text {evaluated }}-d_{\text {real }}\right)\right\}
$$

The origin and the direction of the US beam were therefore expressed by the US probe calibration transformation $T_{U S}^{U S B}$ (see Figure 7). The minimization algorithm was implemented using Matlab ${ }^{\circledR}$ (MathWorks, MA, USA). Residuals are computed as absolute value of the difference between the measured distance and the reconstructed distance with the calibrated parameters.

\section{DATA}

\subsection{US system accuracy}

In order to evaluate the automatic signal detection algorithm and to correct any drift or offset in the acquisition system, we acquired the US signal filling a PMMA box of known shape $(20 \times 100 \mathrm{~mm}$ cavity) with pure water at different temperature (range $5^{\circ}-40^{\circ} \mathrm{C}$ ) and we compared the computed velocities values with the ones computed with Belogol'skii equation $^{20}$, considering a pressure of $1 \mathrm{~atm}$. For each acquired US signal the water temperature was measured with a $\mathrm{Hg}$ thermometer $\left(0.1^{\circ} \mathrm{C}\right.$ precision $)$ and the TOF calculated with the procedure described in 2.4 .

\subsection{US probe calibration}

In order to calibrate the BONUS system, the US probe was free-hand moved over a plastic plane, sunk in water, and 550 poses were acquired, 450 poses were used for "training" and 100 for "testing". The algorithm iteration stops when RMSE on testing data reaches a minimum in order to avoid overfitting on training data. The probe has to be kept perpendicular to the acquired surface, otherwise the reflected echo could miss the transducer. For example, if the reflection surface is $30 \mathrm{~mm}$ distant from transducer, the maximum angle of inclination of a $6 \mathrm{~mm}$ transducer is $5.7^{\circ}$.

The plane equation was computed by fitting a plane on 6 points acquired with an optically tracked palpation probe. Initialization parameters were calculated acquiring the center of the external surface of the US transducer with the same palpation probe. 


\subsection{CT and MRI acquisition}

High resolution 3 CT scans of the phantom (SOMATOM Plus 4, Siemens) and 3 MRI datasets of the phantom were acquired (HARMONY, Siemens) (see Table 2 for acquisition parameters).

Image elaboration was done using Amira ${ }^{\circledR} 4.1$ (Mercury Computer Syst., MA, USA). The 3 CT scans were automatically merged and segmented in order to identify the bone contours. The surface mesh of the phantom was computed and then smoothed with a Garland-Heckbert ${ }^{21}$ filter (with 60 iterations and $\lambda=0.6$ ). The three MR scan acquired (coronal, axial and sagittal) were registered on the CT scan with pair point matching ${ }^{22}$ selecting corresponding anatomical points and maximizing the mutual information ${ }^{23}$. The surface models of the silicone layers were obtained from the MR images segmentation.

Table 2 Parameters list of the acquired CT and MR dataset of the phantom.

\begin{tabular}{ccccc}
\hline & CT dataset & \multicolumn{3}{c}{ MR dataset } \\
Acquisition machine & SOMATOM Plus 4 (Siemens, Germany) & \multicolumn{3}{c}{ HARMONY (Siemens, Germany) } \\
Magnetic field (T) & - & & \multicolumn{2}{c}{1} \\
Echo Time & - & & 686 \\
Repetition Time & 3 & Sagittal & Coronal & Frontal \\
Number of datasets & & (T1) & (T1) & (T1) \\
& & 22 & 20 & 25 \\
Number of images & 268 & $0.6 \times 0.6$ & $0.58 \times 0.58$ & $0.62 \times 0.62$ \\
In-plane resolution & $0.36 \times 0.36$ & $512 \times 416$ & $416 \times 512$ & $512 \times 416$ \\
Number of pixels & $512 \times 512$ & 4 & 4 & 4 \\
Slice thickness (mm) & 1 & 4.8 & 5.2 & 5.2 \\
Slice spacing (mm) & 1 & & & \\
\hline
\end{tabular}

\subsection{Different layers discrimination accuracy}

At first we analyzed the system accuracy in the determination of the different silicone layer thicknesses, considering that the most reflective interface is the cortical bone.

The probe was manually kept over Zones 3, 4 and 5 and the US signal acquired. The thickness of each layer (TL) was therefore computed as:

$$
T L=v_{k} \cdot \frac{T O F}{2}
$$

Where $v_{k}$ is the velocity of the kilicone.

We acquired four anatomical landmarks (anterior cortical point, the epicondyles and the knee center ) by the optically tracked pointer for pair point matching registration with the surface model of the femur. The registration was refined through by surface points acquisition (iterative closest point) $\left(T_{I}^{F}\right)$ (Figure 7). In order to evaluate the accuracy in the silicone layer thickness computation, the calibrated probe was optically tracked $\left(T_{O}^{U S}\right)$. Named $T_{U S}^{U S B}$ the probe calibration matrix, the origin $O_{I}$ and the direction $d_{I}$ of the US beam in the medical images space are then computed as:

$$
\begin{gathered}
O_{I}=T_{I}^{F} \cdot\left(\left(T_{O}^{F}\right)^{-1} \cdot T_{O}^{U S} \cdot T_{U S}^{U S B} \cdot O_{U S B}\right) \\
d_{I}=T_{I}^{F} \cdot\left(\left(T_{O}^{F}\right)^{-1} \cdot T_{O}^{U S} \cdot T_{U S}^{U S B} \cdot d_{U S B}\right)
\end{gathered}
$$

where $O_{U S B}$ and $d_{U S B}$ are the origin and the direction of the US beam in the US probe reference frame and $T_{O}^{F}$ is the transformation between the optical localization system internal reference frame and the femur. In $T_{U S}^{U S B}$ the $\mathrm{Z}$ axis is the US beam direction. 

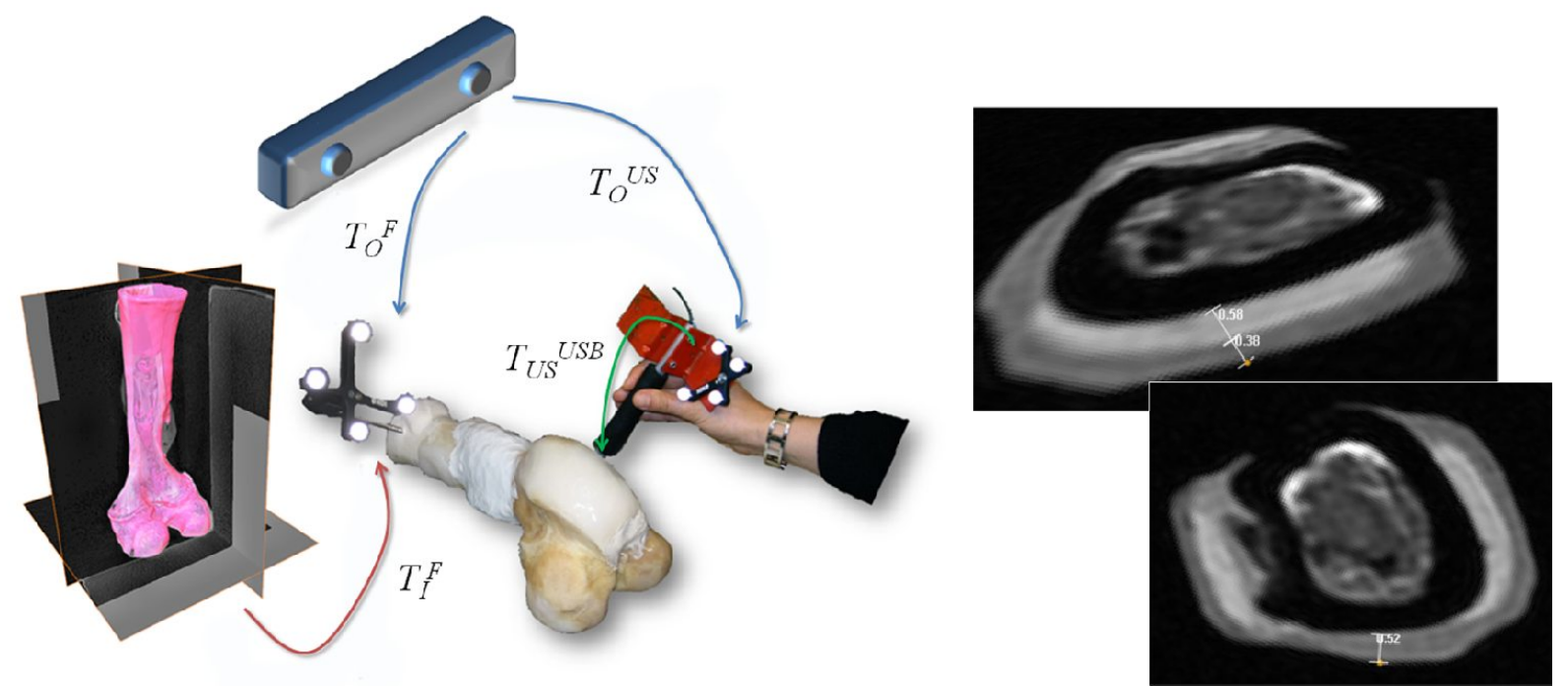

Figure 7 Transformations involved in the system (left). $T_{O}^{F}$ and $T_{O}^{U S}$ are the transformation between the optical localization system internal reference frame and, respectively, the femur and the US probe. $T_{U S}^{U S B}$ is the probe calibration matrix and $T_{I}^{F}$ is the transformation between the physical space and the medical image space. On the right, examples of distance measurements on the MR slices.

$\mathrm{O}_{\mathrm{I}}$ and $\mathrm{d}_{\mathrm{I}}$ allowed measuring the silicone layers thickness on the MR and CT images, using the Distance Measuring tool of Amira ${ }^{\circledR}$ (see Figure 7, right). Such measures were considered as "gold standard" and errors are consequently computed.

\subsubsection{Overall system accuracy}

We evaluated the system accuracy in the detection of points on the exposed and on the silicone covered bone surface. Points on the Zones 8, 3 and 7 of the bone were acquired with the US probe and with a standard pointer (the graphical user interface of the module is shown in Figure 8).

The measured distance $\mathrm{d}_{\mathrm{US}}\left(\mathrm{d}_{\mathrm{US}}=v_{m} . T O F / 2\right.$, where $\mathrm{v}_{\mathrm{m}}$ is the mean of the velocity of the different silicone layers) allows one to determine the $3 \mathrm{D}$ coordinates of the surface point $\mathrm{P}_{\mathrm{I}}$, in the image space:

$$
P_{I}=T_{I}^{F} \cdot\left(\left(T_{O}^{F}\right)^{-1} \cdot T_{O}^{U S} \cdot T_{U S}^{U S B} \cdot O_{U S B}\right)
$$

where

$$
T_{U S B}=\left[\begin{array}{cccc}
1 & 0 & 0 & 0 \\
0 & 1 & 0 & 0 \\
0 & 0 & 1 & d_{U S} \\
0 & 0 & 0 & 1
\end{array}\right]
$$

Coordinates of points acquired with the standard probe are:

$$
P_{p o \text { inter }}=T_{I}^{F} \cdot\left(\left(T_{O}^{F}\right)^{-1} \cdot T_{O}^{U S} \cdot T_{U S}^{U S B} \cdot T_{p o \text { inter }}\right)
$$

where $T_{p o \text { inter }}$ is provided by the VICRA® Tool Tracking software (NDI).

Distances from such points and the closest point on the femur surface are therefore computed with custom written modules in Amira ${ }^{\circledR}$. Statistical analysis of the results was performed and, because the data distribution was not normal, 
non-parametric test (Mann-Whitney U Test) was adopted, using Statistica 7.0 (StatSoft Inc., Tulsa, OK, US). A statistically significant result was given at $\mathrm{p} \leq 0.01$.

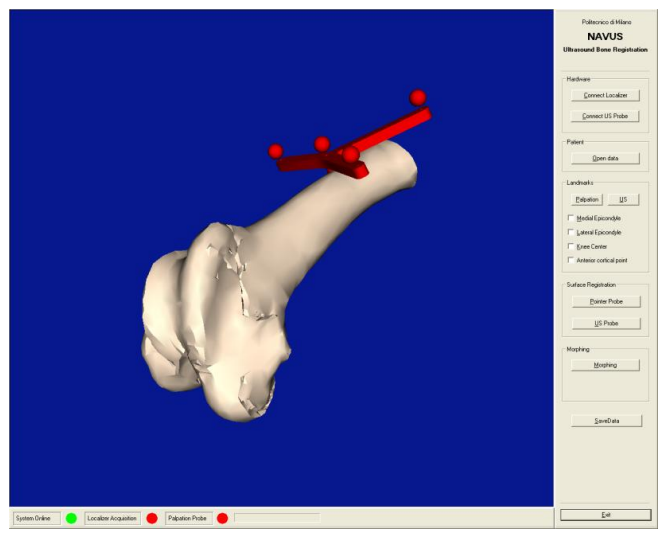

Figure 8 Graphical user interface (GUI) implemented $(\mathrm{C}++)$ to be integrated in a CAOS system, where the surgeon can choose whether to use the traditional pointer probe or the US probe to intra-operatively acquired anatomical points of interest.

\section{RESULTS}

\subsection{Signal elaboration}

The acquired velocity values are well aligned with the values found in literature in the considered range of temperatures $\left(5-40^{\circ} \mathrm{C}\right)$. The RMSE between the acquired and the tabulated velocity values is $0.87 \mathrm{~m} / \mathrm{s}$ over 77 acquired samples.

\subsection{Materials characterization}

The materials were characterized computing the US speed in $20 \mathrm{~mm}$ length PMMA boxes. In Table 3 median values, obtained in 9 acquisitions are reported. SYL, SARA and BOT dispersion values are within the measuring errors $(0.87 \mathrm{~m} / \mathrm{s}$ RMS), while BOW values are spread, due to the high signal attenuation.

Table 3 Results on materials characterization.

\begin{tabular}{lllllll}
\hline & $\begin{array}{l}\text { Velocity } \\
\text { median value }\end{array}$ & $-25^{\text {th }}$ percentile & $75^{\text {th }}$ percentile & $\begin{array}{l}\text { Attenuation } \\
(\mathrm{dB} / \mathrm{cm} \cdot \mathrm{MHz})\end{array}$ & $\begin{array}{l}\text { Density } \\
\left(\mathrm{kg} / \mathrm{m}^{3}\right)\end{array}$ & $\begin{array}{l}\text { Temperature } \\
\left({ }^{\circ} \mathrm{C}\right)\end{array}$ \\
SYL & 1053.87 & 0.55 & 1.04 & & 1020 & 27 \\
SARA & 1068.18 & 0.57 & 1.14 & 0.56 & 98.8 & 27 \\
BOT & 1027.75 & 0.79 & 1.85 & & - & 28 \\
BOW & 1318.82 & 7.13 & 5.47 & & - & 27 \\
\hline
\end{tabular}

\subsection{Calibration residuals}

The calibration algorithm stopped when the RMSE of the testing data reached a minimum (after 684 iterations). The RMSE of residuals on testing data is $0.37 \mathrm{~mm}$.

\subsection{System accuracy}

The differences between the silicone layers thickness measured by the US system and analyzing the medical images are reported in Figure 9. In Zone 3, where a single silicone layer is present, errors are less than $0.25 \mathrm{~mm}$, comparable with the axial resolution of the US transducer. Remarkable results are also present in Zone 5, where the system is able to discriminate between two types of silicone with high accuracy (less than $0.1 \mathrm{~mm}$ ). Higher errors corresponding to Zone 4 are greater than $2 \mathrm{~mm}$ in most superficial layer, thus affecting the measure of the total thickness. 


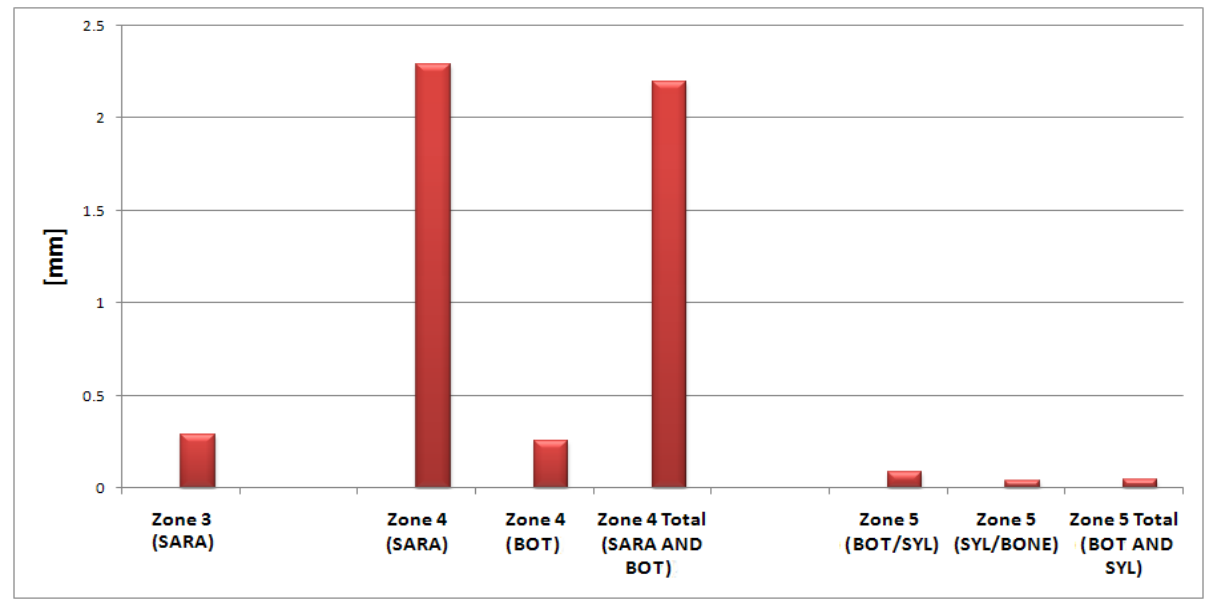

Figure 9 Absolute differences between the silicone layers thickness measured by the US system and analyzing the medical images in zones 3, 4 and 5 .

Registration error between physical space and image space (in terms of Fiducial Registration Error, FRE ${ }^{17}$ ) is $0.15 \mathrm{~mm}$ as median value. On exposed surfaces (Bone Interface), the tracked pointer shows a median distance value of $0.14 \mathrm{~mm}$, comparable with the accuracy of the optical system and with the registration error. Such value is significantly better than the US probe value $(0.21 \mathrm{~mm}$ median value) which is still acceptable. This is visually confirmed by Figure 10 , where US points and palpated points are superimposed. In the phantom regions covered by silicone, the US probe behaves significantly better ( $\max$ median value $1.2 \mathrm{~mm}$ ) compared to the pointer.
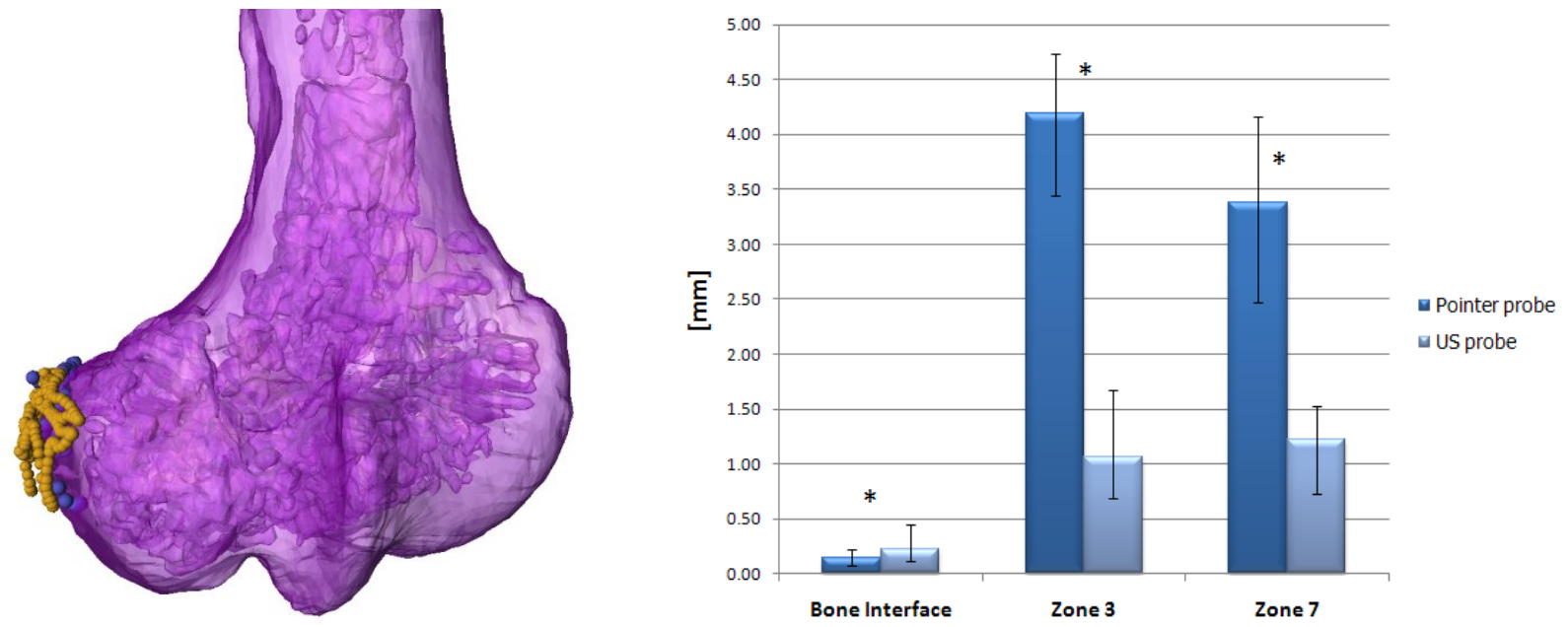

Figure 10 Acquired US points (blue) and palpated points (yellow) on the bone surface (on the left). Distance values (median, $25^{\text {th }}$ and $75^{\text {th }}$ percentiles) from the gold standard (CT) bone surface for points acquired with both US probe and palpation pointer.

\section{CONCLUSIONS}

Anatomical landmarks and bone surface acquisition is straightforward in CAOS procedures in order to correctly align prostheses components. Several works showed that intraobserver errors in landmark acquisition can reach maximum values of $7 \mathrm{~mm}$ and interobserver errors can raise up to $20 \mathrm{~mm}^{7}$. Errors can be due to the operator inaccuracy in choosing an ideal landmark and also to the soft tissue layer interposition, in case of anatomical points not exposed during the surgery. As consequence, US systems, which allow transcutaneous acquisition of bone surface points, can be identified as a non-invasive pointer for CAOS systems.

Differently from B-mode based systems, A-mode signal detection allows for real-time surface points detection since there is no need of images segmentation (the signal acquisition and processing was implemented on the FPGA board). 
Our system proved to be real-time and the acquired probe pose, detected by the localization system, was perfectly synchronized with the received US signal.

In order to calibrate US A-mode transducers Lewis ${ }^{14}$ replaced the US transducer with an adjustable length pointer with a spherical tip and pivoted the tool around a divot that exactly fitted the tip, so that the optical system could see the probe in many orientations while the center of the spherical tip remained fixed. The procedure is time consuming and difficult to realize in case of recalibration. Residuals errors are not reported, but they were strongly affected by the accuracy of the transducer repositioning after the pivoting. The calibration procedure that we propose is easy, repeatable and less time consuming. It requires US probe free-hand movements on a plane, whose coordinates are known in the optical localizator reference system. Residual errors have the same order of magnitude of the TRE of the optically tracked pointer.

As first step we wanted to determine the accuracy in the computation of the TOF, provided the correct detection of the reflection peak of the signal. Comparing the tabulated values for the speed of sound in distilled water at given temperature and pressure values with the ones computed with our US system, we found our results coherent with the tabulated values, thus excluding any offset (mean difference $-0.42 \mathrm{~m} / \mathrm{s} \pm 0.77 \mathrm{~m} / \mathrm{s}$ ).

Soft-tissue mimicking materials are useful in pre-clinical studies since they have similar mechanical properties (US propagation speed and attenuation) of real tissues and they can be easily collected, maintaining their physical properties in time so that experiments can be repeatedly performed. Kondo ${ }^{24}$ tested the behavior of agar, oil gel and polyurethane gel in terms of speed of sound and attenuation. While speed of sound is comparable with the speed of sound in human soft tissue $(1500 \mathrm{~m} / \mathrm{s})$, the attenuation coefficient is quite different $(1.12-0.13 \mathrm{vs} 0.7 \mathrm{~dB} / \mathrm{cm} \cdot \mathrm{MHz}$ for agar and polyurethane gel, and 0.4-1.8 for oil gel). Although, silicone sealant we used showed a lower speed of sound (around $1030-1300 \mathrm{~m} / \mathrm{s})$, we found an attenuation similar to the one of soft tissue $(0.56 \mathrm{~dB} / \mathrm{cm} \cdot \mathrm{MHz}$ for SARA). Also, one of the most important problem of soft tissue mimicking materials is their rapid decay, which compromises test repeatability. Silicone material maintains longer its mechanical properties than agar.

Different layers of different kind of silicones were reproduced on a bovine bone phantom in order to evaluate the system behavior (in terms of beam reflection and refraction) at interfaces with similar acoustic impedance, mimicking the different soft tissue of the joints (fat, muscle, tendons). Even if the working conditions in the operating room would be quite different, testing the system with the bone phantom is straightforward before starting the clinical tests.

Comparing the detected thickness of the silicone layers with the US system and with the manual selection on the MR images with the Amira SW, one can see that the US system allows the detection of the thickness of the silicone layer with high accuracy. This is true either when the layer is formed by a single silicone type, either when there are two layers. Nevertheless, the inaccuracies reported for Zone 4 are probably due to the gel material which was interpose in order to optimize the acoustic coupling.

Accuracy tests of the whole system showed that the US probe acquisition behaves significantly better (max median value $1.2 \mathrm{~mm}$ vs. $4.2 \mathrm{~mm}$ ) compared to the pointer acquisition, confirming that BONUS system can considerably increase accuracy of registration and biomechanical point computation.

Future developments will focus on signal processing for real time US velocity correction to improve accuracy on distance detection. This can be done, for example, by the investigation of US elastography for different tissue characterisation and different US velocity association for each tissue. Also, the system will equip it with a friendly graphical interface which will show to the operator the current pose of the probe in the femoral reference frame and the uncertainness of the point localization, due to tracking errors. This will allow the operator to check the perpendicularity between the US beam and the reflection surface underneath. Then, clinical tests will be performed for in vivo assessment of system accuracy in real situations.

\section{REFERENCES}

[1] Haaker, R. G., Stockheim, M., Kamp, M., Proff, G., Breitenfelder, J., Ottersbach, A., “Computer-assisted navigation increases precision of component placement in total knee arthroplasty.", Clin. Orthop. Relat. Res., 433, 152-9 (2005). 
[2] De Momi, E., Cerveri, P., Ferrigno, G., [User Centered Design for Medical Visualization], Feng Dong, Gheorghita Ghinea and Sherry Y. Chen, Brunel University, UK, 205-222 (2008).

[3] Stöckl, B., Nogler, M., Rosiek, R., Fischer, M., Krismer, M., Kessler, O., "Navigation improves accuracy of rotational alignment in total knee arthroplasty", Clin. Orthop. Relat. Res., 426, 180-6 (2004).

[4] Barratt, D. C., Chan, C. S., Edwards, P. J., Penney, G. P., Slomczykowski, M., Carter., T. J., Hawkes, D. J., "Instantiation and registration of statistical shape models of the femur and pelvis using 3D ultrasound imaging", Med. Image Anal., 12(3), 358-74 (2008)

[5] Talib, H., Rajamani, K., Kowal, J., Nolte, L. P., Styner, M., Ballester, M. A., “A comparison study assessing the feasibility of ultrasound-initialized deformable bone models",. Comp. Aid. Surg. 10 (5-6), 293-299 (2005)

[6] Fleute, M., Lavallee, S., Julliard, R., "Incorporating a statistically based shape model into a system for computerassisted anterior cruciate ligament surgery",. Med. Image Anal., 3209-3222 (1999).

[7] Yau, W. P., Leung, A., Liu, K. G., Yan, C. H., Wong, L. L., Chiu, K. Y., "Intraobserver Errors in Obtaining Visually Selected Anatomic Landmarks During Registration Process in Nonimage-based Navigation-assisted Total Knee Arthroplasty", J. Arthroplasty 22(8), 1150-1161 (2007)

[8] Robinson, M., Eckhoff, D. G., Reinig, K. D., Bagur, M. M., Bach, J. M., "Variability of landmark identification in total knee arthroplasty", Clin. Orthop. Relat. Res., 442, 57-62 (2006)

[9] Jain, A., Taylor, R., "Understanding bone responses in B-mode ultrasound images and automatic surface extraction using a Bayesian probabilistic framework.", Proc. SPIE, 131-142 (2004).

[10] Jaramaz, B., Nikou, C., Cavalier, B., Blendea, S., La Barca, R., Di Gioia, A. M., "Experimental validation of ultrasound registration of long bones", Proceedings of the 3rd Annual Meeting of CAOS International, 160-161 (2003).

[11] Barratt, D. C., Penney, G., Chan, C. S., Slomczykowski, M., Carter, T. J., Edwards, P. J., Hawkes, D. J., "Selfcalibrating 3D-ultrasound-based bone registration for minimally invasive orthopedic surgery", Med. Image Anal., 10(3), 385-95 (2006)

[12] Calvin, R., Maurer, Jr., Ronald, P., Gaston, Derek, L. G., Hill, Michael, J., Gleeson, M., Graeme Taylor, Michael, R., Fenlon, Philip J., Edwards, David, J., Hawkes, "AcouStick: A Tracked A-Mode Ultrasonography System for Registration in Image Guided Neurosurgery”, Stereotact. Funct. Neurosurg., 72(2-4), 143-144 (1999)

[13] Schreiner, S., Galloway, R. L. Jr., Lewis, J. T., Bass, W. A., Muratore, D. M., "An ultrasonic approach to localization of fiducial markers for interactive, image-guided neurosurgery--Part II: Implementation and automation", IEEE Trans. Biomed. Eng., 45(5), 631-41 (1998).

[14] Lewis, J. T., Galloway R. L., and Schreiner, S., “An Ultrasonic Approach to Localization of Fiducial Markers for Interactive, Image-Guided Neurosurgery”, IEEE Trans. Biom. Eng., 45(5), 620-641 (1998)

[15] Heger, S., Portheine, F., Ohnsorge, J. A. K., Schkommodau, E. and Radermacher, K., "User-interactive registration of bone with A-mode ultrasound", IEEE Eng. in Med. and Biology magazine, 24(2), 85-95 (2005)

[16] Heger, S., Mumme, T., Sellei, R., De La Fuente, M., Wirtz, D. C., Radermacher, K., "A-mode ultrasound-based intra-femoral bone cement detection and 3D reconstruction in RTHR.”, Comput. Aided Surg., 12(3), 168-75 (2007).

[17] Fitzpatrick, J. M., West, J. B., "Distribution of target registration errorin rigid-body point-based registration.”, IEEE Trans. Med. Imag., 20, 917-927 (2001).

[18] Levenberg, K., "A Method for the Solution of Certain Non-linear Problems in Least Squares", Quarterly of Applied Mathematics, 2(2), 164-168 (1944).

[19] Marquardt, D., "An Algorithm for Least-Squares Estimation of Nonlinear Parameters", SIAM Journal on Applied Mathematics, 11, 431-441 (1963).

[20] Belogol'skii, V. A., Sekoyan, S. S., Samorukova, L. M., Stefanov, S. R., Levtsov, V. I., "Pressure dependence of the sound velocity in distilled water", Measurement Techniques, 42(4), 406-413 (1999).

[21] Garland, M., Heckbert, P. S., "Surface Simplification Using Quadric Error Metrics", Computer Graphics, 31, 209216 (1997).

[22] Horn, B. K. P., "Closed-form solution of absolute orientation using unit quaternions," J. Opt. Soc. Am. A., 4, 629 (1987).

[23] Viola, P., and Wells, III W. M., “Alignment by maximization of mutual information”, Int. J. Computer Vision, 24 (2), 137-154 (1997).

[24] Kondo, T., Kitatuji, M., Kanda, H., "New Tissue Mimicking Materials for Ultrasound Phantoms", Ultrasonics Symposium, IEEE, 3, 1664 - 1667 (2005) 